\title{
Performance Assessments and Comparisons of S-NPP and NOAA-20 (JPSS-1) VIIRS On-orbit Calibration
}

\author{
Xiaoxiong Xiong ${ }^{1}$, Amit Angal ${ }^{2}$, James Butler ${ }^{1}$, Hongda Chen ${ }^{2}$, Kwofu Chiang ${ }^{2}$, \\ Ning Lei ${ }^{2}$, Yonghong $\mathrm{Li}^{2}$, and Kevin Twedt ${ }^{2}$ \\ ${ }^{1}$ Sciences and Exploration Directorate, NASA GSFC, Greenbelt, MD 20771, USA \\ ${ }^{2}$ Science Systems and Applications Inc., 10210 Greenbelt Rd, Lanham, MD 20706, USA
}

\begin{abstract}
The first Visible Infrared Imaging Radiometer Suite (VIIRS) instrument has been in operation for more than 6 years on-board the S-NPP satellite and the second instrument, with the same design and performance requirements, was launched in November, 2017 on-board the JPSS-1 satellite (named NOAA-20 after reaching its orbit) and is currently in normal operation conditions. This paper provides a brief description of VIIRS on-orbit calibration and characterization activities and presents performance assessments and comparisons of S-NPP and NOAA-20 VIIRS using data collected from their on-board calibrators (OBC) and regularly scheduled lunar observations. Results show that NOAA-20 VIIRS is performing as well or better than S-NPP VIIRS in all of the key performance metrics. The NOAA-20 reflective solar bands, including the day-night band, have experienced less than $1 \%$ change in gain in the first 250 days since launch and did not suffer from the contamination related rapid degradation experienced by S-NPP VIIRS. Some of the NOAA20 thermal emissive bands had larger than expected gain degradation after launch due to ice buildup on the dewar window of the long-wave IR focal plane assembly but a mid-mission outgassing operation was able to restore their gains and maintain stable behavior. Though this study is focused on the sensor's key performance parameters, such as detector responses (gains), signal-to-noise ratios, and noise-equivalent temperature differences, challenges identified and lessons learned through different phases of on-orbit calibration and characterization are also discussed.
\end{abstract}

Keywords: VIIRS, S-NPP, NOAA-20, calibration, on-board calibrators, DNB, Moon

\section{INTRODUCTION}

Two nearly identical VIIRS instruments are currently operated in the same orbit, one on-board the Suomi National Polar-orbiting Partnership (S-NPP) satellite launched in October, 2011 and the other on-board the NOAA-20 (N-20) satellite (formerly the JPSS-1) launched in November, 2017, with N-20 orbiting about 50 minutes ahead of S-NPP. The VIIRS is a scanning radiometer that uses a rotating telescope assembly (RTA), coupled with a two-sided half-angle mirror (HAM). It collects data in 22 spectral bands: 14 reflective solar bands (RSB), 7 thermal emissive bands (TEB), and 1 day/night band (DNB), covering wavelengths from visible (VIS) to long-wave infrared (LWIR). DNB observations can be made in 3 gain stages, thus capable of making measurements over a very large dynamic range ${ }^{1-4}$. For reference purposes, several key design parameters of VIIRS spectral bands, such as their wavelengths, typical radiances (or temperatures), and specified signal-to-noise ratios (SNR) or noise-equivalent temperature differences (NEdT), are listed in Table 1. Also listed in Table 1 are the on-orbit SNR and NEdT for both S-NPP and N-20 VIIRS at their mission beginning and present. More details on instrument performance will be discussed in section 4 of this paper. 
Table 1. VIIRS spectral band design specifications and on-orbit SNR or NEdT (radiance unit: $\mathrm{W} / \mathrm{m}^{2} / \mu \mathrm{m} / \mathrm{sr}$, temperature unit: K). M1 specified Ltyp was changed from 0.12 to $1.0 \mathrm{~W} / \mathrm{m}^{2} / \mu \mathrm{m} / \mathrm{sr}$.

\begin{tabular}{|c|c|c|c|c|c|c|c|c|}
\hline $\begin{array}{l}\text { Band } \\
\text { No. }\end{array}$ & $\begin{array}{l}\text { Spectral Range } \\
\qquad(\mu \mathrm{m})\end{array}$ & $\begin{array}{l}\text { Band } \\
\text { Gain }\end{array}$ & $\begin{array}{c}\mathrm{L}_{\text {typ }} \text { or } \mathrm{T}_{\text {typ }} \\
\text { Spec }\end{array}$ & $\begin{array}{l}\text { SNR or } \\
\text { NEdT } \\
\text { Spec }\end{array}$ & $\begin{array}{c}\text { S-NPP } \\
\text { SNR or } \\
\text { NEdT } \\
(02 / 06 / 12) \\
\end{array}$ & $\begin{array}{c}\text { S-NPP } \\
\text { SNR or } \\
\text { NEdT } \\
(06 / 21 / 18) \\
\end{array}$ & $\begin{array}{c}\text { N-20 } \\
\text { SNR or } \\
\text { NEdT } \\
(01 / 13 / 18)\end{array}$ & $\begin{array}{c}\text { N-20 } \\
\text { SNR or } \\
\text { NEdT } \\
(06 / 21 / 18)\end{array}$ \\
\hline \multirow{2}{*}{ M1 } & \multirow{2}{*}{$0.402-0.422$} & High & 44.9 & 352 & 594.9 & 555.0 & 637.1 & 647.3 \\
\hline & & Low & 155 & 316 & 1064 & 990.5 & 1100 & 1117 \\
\hline \multirow{2}{*}{ M2 } & \multirow{2}{*}{$0.436-0.454$} & High & 40 & 380 & 585.2 & 570.0 & 566.6 & 571.5 \\
\hline & & Low & 146 & 409 & 1055 & 1034 & 982.8 & 1009 \\
\hline \multirow{2}{*}{ M3 } & \multirow{2}{*}{$0.478-0.498$} & High & 32 & 416 & 636.5 & 629.0 & 692.6 & 693.4 \\
\hline & & Low & 123 & 414 & 1030 & 994.4 & 1052 & 1061 \\
\hline \multirow{2}{*}{ M4 } & \multirow{2}{*}{$0.545-0.565$} & High & 21 & 362 & 543.0 & 536.5 & 547.7 & 555.7 \\
\hline & & Low & 90 & 315 & 856.8 & 847.4 & 840.4 & 852.1 \\
\hline I1 & $0.600-0.680$ & Single & 22 & 119 & 213.0 & 195.2 & 224.6 & 224.2 \\
\hline \multirow{2}{*}{ M5 } & \multirow{2}{*}{$0.662-0.682$} & High & 10 & 242 & 331.5 & 289.9 & 389.6 & 385.5 \\
\hline & & Low & 68 & 360 & 626.4 & 626.8 & 766.8 & 762.5 \\
\hline M6 & $0.739-0.754$ & Single & 9.6 & 199 & 362.2 & 311.8 & 418.9 & 412.9 \\
\hline I2 & $0.846-0.885$ & Single & 25 & 150 & 258.0 & 196.8 & 281.7 & 282.0 \\
\hline \multirow{2}{*}{ M7 } & \multirow{2}{*}{$0.846-0.885$} & High & 6.4 & 215 & 447.2 & 340.8 & 531.5 & 527.8 \\
\hline & & Low & 33.4 & 340 & 622.2 & 433.5 & 713.3 & 709.6 \\
\hline M8 & $1.230-1.250$ & Single & 5.4 & 74 & 229.4 & 165.1 & 323.8 & 320.0 \\
\hline M9 & $1.371-1.386$ & Single & 6 & 83 & 231.6 & 182.4 & 298.2 & 296.1 \\
\hline I3 & $1.580-1.640$ & Single & 7.3 & 6 & 152.9 & 131.4 & 172.5 & 172.8 \\
\hline M10 & $1.580-1.640$ & Single & 7.3 & 342 & 595.1 & 518.5 & 668.6 & 654.2 \\
\hline M11 & $2.225-2.275$ & Single & $0.12 *$ & 10 & 22.1 & 20.4 & 200.0 & 198.8 \\
\hline I4 & $3.550-3.930$ & Single & $270(\mathrm{~K})$ & $2.5(\mathrm{~K})$ & 0.408 & 0.409 & 0.381 & 0.385 \\
\hline M12 & $3.660-3.840$ & Single & $270(\mathrm{~K})$ & $0.396(\mathrm{~K})$ & 0.128 & 0.118 & 0.099 & 0.100 \\
\hline \multirow{2}{*}{ M13 } & \multirow{2}{*}{$3.973-4.128$} & High & $300(\mathrm{~K})$ & $0.107(\mathrm{~K})$ & 0.042 & 0.040 & 0.037 & 0.037 \\
\hline & & Low & $380(\mathrm{~K})$ & $0.423(\mathrm{~K})$ & N/A & $\mathrm{N} / \mathrm{A}$ & $\mathrm{N} / \mathrm{A}$ & N/A \\
\hline M14 & $8.400-8.700$ & Single & $270(\mathrm{~K})$ & $0.091(\mathrm{~K})$ & 0.053 & 0.055 & 0.047 & 0.047 \\
\hline M15 & $10.263-11.263$ & Single & $300(\mathrm{~K})$ & $0.07(\mathrm{~K})$ & 0.029 & 0.027 & 0.023 & 0.023 \\
\hline I5 & $10.500-12.400$ & Single & $210(\mathrm{~K})$ & $1.5(\mathrm{~K})$ & 0.384 & 0.400 & 0.307 & 0.311 \\
\hline M16 & $11.538-12.488$ & Single & $300(\mathrm{~K})$ & $0.072(\mathrm{~K})$ & 0.028 & 0.029 & 0.031 & 0.031 \\
\hline
\end{tabular}

Like its predecessor MODIS, currently operated on-board the NASA's EOS Terra and Aqua spacecraft, the VIIRS on-orbit calibration is performed using a set of on-board calibrators (OBCs), which include a solar diffuser (SD) coupled with an attenuation screen, a solar diffuser stability monitor (SDSM) operated as a ratioing radiometer to track SD on-orbit degradation, and a blackbody that can be controlled at select temperatures. A space view (SV) port provides a view of deep space for use as a dark background reference 
and a way to make observations of the moon. Figure 1 shows a diagram of the VIIRS scan cavity and OBCs. Both MODIS and VIIRS instruments were built by the same instrument vendor. As expected, VIIRS OBCs were designed and operated based on the lessons and experience learned from MODIS.

This paper provides a brief description of VIIRS on-orbit operation and calibration activities, especially those performed during the initial intensive calibration and validation (ICV) phase, followed by the calibration methodologies for both RSB and TEB. The OBC functions for both instruments and their on-orbit performance are examined and illustrated. Data collected from the OBCs and regularly scheduled lunar observations are used to derive the sensor's key performance parameters. Preliminary results from N-20 VIIRS are compared with results from S-NPP. As illustrated in this paper, the overall performance of N-20 VIIRS is slightly better than S-NPP. Initial ice buildup on the dewar widow of the LWIR focal plane assemblies (FPA), which caused degradation of TEB responses (gains), was eliminated via a mid-mission outgassing (MMOG). Unlike S-NPP, the N-20 RSB responses have been very stable. Lessons and experiences from S-NPP have directly contributed to the success of N-20 VIIRS on-orbit calibration.
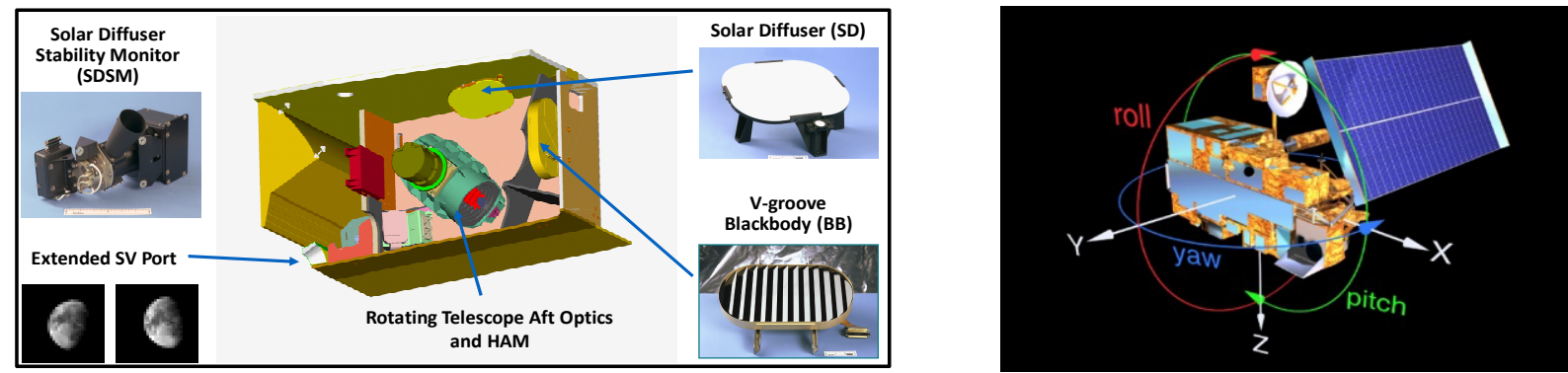

Figure 1. VIIRS scan cavity and its on-board calibrators (OBC) (left) and illustration of spacecraft maneuvers (right).

\section{VIIRS ON-ORBIT OPERATION AND CALIBRATION ACTIVITIES}

During the ICV period of both instruments, a number of special tests to assess sensor at launch performance were conducted. Three different types of maneuvers (shown in the right panel of Figure 1) in support of VIIRS on-orbit calibration and validation effort are a key part of the ICV activities: (1) roll maneuvers for lunar observations to monitor the RSB calibration stability, (2) yaw maneuvers to validate the SD BRF and solar attenuation screen (SAS) transmission, as well as SDSM screen transmission, and (3) a pitch maneuver to validate the TEB response versus scan-angle (RVS). Tables 2 and 3 provide a brief summary of the key operation events and calibration activities.

Early in the S-NPP mission, an abnormal degradation was observed in the NIR and SWIR detector responses. A number of special tests were performed thereafter to identify and characterize the root cause of the degradation that was later confirmed to be due to the contamination of RTA mirror coatings that occurred pre-launch $^{5-7}$. The largest impact of this degradation was observed in the bands M6-M9 and I2. In the case of $\mathrm{N}-20$, accelerated degradation in detector gains was observed shortly after launch in the LWIR bands (I5, M15 and M16). Following a number of diagnostic tests, including RTA stow and electronic calibration, to trend the degradation behavior (especially its spectral dependence), the likely cause of the degradation was believed to be due to the ice buildup on the dewar window of the LWIR FPA. A MMOG operation performed in March, 2018 was able to recover the gain decreases that resulted from the ice-buildup. Both S- 
NPP and N-20 VIIRS lunar observations, performed via a spacecraft roll maneuver and sensor data sector rotation, are collected in a fixed high gain mode near-monthly (7-9 times a year). The S-NPP VIIRS lunar observations are typically collected in aggregation zone 3 . In contrast, the N-20 VIIRS lunar observations are collected in aggregation zone 1, with the exception of the first lunar event on December 29, 2017, which was collected in aggregation zone 2 . The on-board bowtie deletion is disabled during its lunar calibration events.

Table 2. Key operation and calibration events of S-NPP VIIRS

\begin{tabular}{|c|l|l|}
\hline Event \# & \multicolumn{1}{|c|}{ Date } & \multicolumn{1}{c|}{ Event Description } \\
\hline 1 & $10 / 28 / 2011$ & Suomi-NPP Launch \\
2 & $11 / 08 / 2011$ & VIIRS Turned On \\
3 & $11 / 21 / 2011$ & Nadir Door Open \\
4 & $12 / 09 / 2011$ & RTA stow to investigate RSB abnormal \\
5 & $01 / 04 / 2012$ & $1^{\text {st }}$ Lunar Calibration (Roll Maneuver) \\
6 & $01 / 18 / 2012$ & Cryo-Cooler Door Open \\
7 & $02 / 06 / 2012$ & $1^{\text {st }}$ OBC Blackbody Warm-Up Cool-Down \\
8 & $02 / 15 / 2012$ & Yaw Maneuver (One Time Event) \\
9 & $02 / 20 / 2012$ & Pitch Maneuver (One Time Event) \\
\hline
\end{tabular}

Table 3. Key operation and calibration events of NOAA-20 VIIRS

\begin{tabular}{|c|c|l|}
\hline Event \# & Date & \multicolumn{1}{|c|}{ Event Description } \\
\hline 1 & $11 / 18 / 2017$ & NOAA-20 Launch \\
2 & $11 / 28 / 2017$ & VIIRS Turned On \\
3 & $12 / 13 / 2017$ & Nadir Door Open \\
4 & $12 / 29 / 2017$ & $1^{\text {st }}$ Lunar Calibration (Roll Maneuver) \\
5 & $01 / 03 / 2018$ & Cryo-Cooler Door Open \\
6 & $01 / 10 / 2018$ & $1^{\text {st }}$ OBC Blackbody Warm-Up Cool-Down \\
7 & $01 / 25 / 2018$ & Yaw Maneuver (One Time Event) \\
8 & $01 / 31 / 2018$ & Pitch Maneuver (One Time Event) \\
9 & $03 / 12 / 2018$ & Mid-mission Outgassing (One Time Event) \\
\hline
\end{tabular}

The on-board blackbody is controlled nominally at a constant temperature for both S-NPP and N-20. Periodic blackbody warm-up and cool-down (WUCD) events, with each taking about 2 days to complete, have been carried out every three months for both instruments. All TEB detectors have shown excellent stability. A recent decision was made to reduce the frequency of future BB WUCD from a quarterly to yearly basis. VIIRS SD calibration data is available every orbit. The SDSM is currently operated on a daily basis for N-20 and three times per week for S-NPP VIIRS. 


\section{ON-ORBIT CALIBRATION METHODOLOGIES}

The VIIRS RSB on-orbit calibration is reflectance-based using observations of the SD, which is illuminated by the sun once every orbit through a fixed attenuation screen. The bidirectional reflectance distribution function (BRDF) of the SD and the transmission of the attenuation screen were measured prior to launch over a range of different illumination angles. After launch, the SD BRDF degrades, likely caused by the solar UV radiation, and this degradation is tracked using the SDSM. The SDSM acts as a ratioing radiometer, making alternate views of the SD, a dark background, and the Sun through a separate fixed attenuation screen on consecutive RTA scans. The ratio of the SDSM detector response when viewing the SD to the response when viewing the Sun, with corrections for the screen transmission and SD BRDF, is denoted as the $\mathrm{H}$-factor. The H-factor is tracked on-orbit for each of the 8 SDSM detectors, which cover the wavelengths of the VIS/NIR bands, and the results are extrapolated to the wavelengths of the SWIR bands ${ }^{3,8-9}$.

The radiance viewed by the VIIRS RSB is calculated from a polynomial function of the backgroundsubtracted detector digital count. The polynomial coefficients were measured pre-launch and an overall multiplicative factor, the F-factor, is used to track the time-dependent on-orbit changes. The F-factor is calculated every orbit from the SD observation, taking into account the solar radiance, the SD screen transmission, the BRDF of the SD and its degradation as tracked by the $\mathrm{H}$-factor, and the relative spectral response of each detector. The inverse of the SD F-factors normalized to the start of the mission can be considered the on-orbit gain change of the RSB. A similar SD and SDSM calibration approach is applied to the DNB low gain stage (LGS) using a simple linear algorithm. The DNB high gain and mid gain stages (HGS and MGS) are calibrated using ratios of HGS to MGS and MGS to LGS, respectively ${ }^{10-11}$.

The TEB calibration converts the background corrected digital response of a detector to the corresponding at sensor aperture radiance using a quadratic model. The coefficients in the model were derived pre-launch. In addition, there is a scale factor (called F-factor) on top of the quadratic model, which is determined on a scan-by-scan basis from the observations of the on-orbit blackbody (BB) and the space view (SV) port. The at-detector radiance when VIIRS views the $\mathrm{BB}$ (or SV) is the sum of the $\mathrm{BB}$ (or SV) radiance and the radiance from a number of emissive sources along the optical path (e.g. RTA, HAM, etc.). The effective BB path radiance is the radiance from the BB path after subtracting the radiance from the SV path, in which the RVS is considered and the contributions from the emissive sources are weighted. The calibration coefficients, RVS, and the temperature dependent radiance of all emissive sources are determined on a perscan basis via on-orbit telemetry measurements and look-up-tables (LUTs). The ratio of the BB path radiance and the radiance from the quadratic model is the F-factor that will be used in the Earth view (EV) data calibration $^{3,12}$. Currently, the TEB LUTs are characterized based on the prelaunch data analysis.

The VIIRS lunar observations are made through its SV port, which is an extension of the EV port. Due to the spacecraft operation and roll angle constraints, the lunar observations are typically made with phase angles between $-51.5^{\circ}$ to $-50.5^{\circ}$. A data sector rotation is performed to store the lunar data in the EV data sector. In some instances, such as June 23, 2018 lunar event, the Moon is directly observed in the SV port therefore bypassing the need for a roll maneuver. To date, S-NPP VIIRS has performed nearly 50 lunar observations and N-20 VIIRS 7 observations. The lunar observations can be used to derive the instrument relative gains (inverse of the F-factors) using the ROLO model predicted lunar irradiance to correct for the lunar viewing geometry differences ${ }^{13}$. 


\section{ASSESSMENTS AND COMPARISONS OF S-NPP AND N-20 VIIRS PERFORMANCE}

Figure 2 shows on-orbit degradation in SDSM detector gains. For each SDSM detector, the gain is calculated from its digital count (dc) with background subtracted and corrections applied for SDSM screen transmittance and the VIIRS-Sun distance. The SDSM detector gain in Figure 2 is normalized to its value at the first available orbit: 154 for the S-NPP and 166 for the N-20. The SDSM detector gains for both the SNPP and the N-20 decrease over time. The largest decrease occurs for the detector at the longest wavelength, i.e. detector 8 at $0.93 \mu \mathrm{m}$. By the time 210 days after the respective satellite launch, the detector 8 gain has decreased $18 \%$ for S-NPP and 17\% for N-20. In general, the SDSM detector gain continues to decrease over time. With more than 6 years of on-orbit operation, the S-NPP SDSM gain degradation has reached $40 \%$ at $0.93 \mu \mathrm{m}, 24 \%$ at $0.86 \mu \mathrm{m}$, and less than $10 \%$ at other shorter wavelengths. The root cause of this wavelength dependent degradation is likely the radiation damage to the detectors by the high energy particles in the space environment. Similar degradation is also observed in MODIS SDSM detector gains ${ }^{14}$.
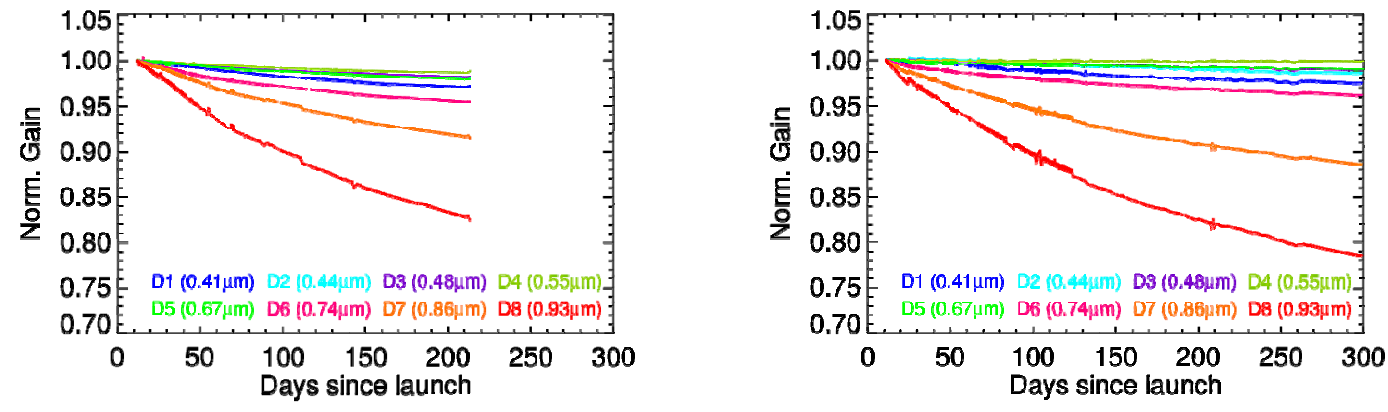

Figure 2. Normalized gains of the SDSM detectors for N-20 (left) and S-NPP (right) VIIRS.
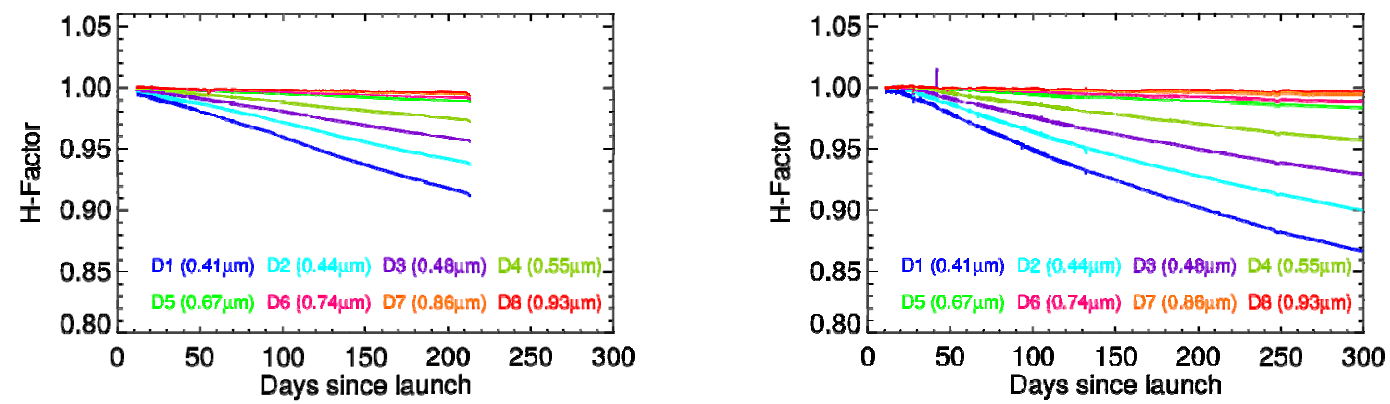

Figure 3. On-orbit SD degradation (H-factors) for N-20 (left) and S-NPP (right) VIIRS.

In general, the H-factors for both the S-NPP and the N-20 VIIRS instruments decrease over time, as shown in Figure 3, at nearly the same rates. The H-factors decrease more at shorter wavelengths. After 210 days since launch, the $\mathrm{H}$-factor has decreased about $10 \%$ at $0.41 \mu \mathrm{m}$, for both S-NPP and N-20 SD. With more than 6 years of on-orbit operation, the S-NPP VIIRS SD degradation has reached $37 \%$ at $0.41 \mu \mathrm{m}, 16 \%$ at $0.55 \mu \mathrm{m}$, and less than $8 \%$ at wavelengths above $0.67 \mu \mathrm{m}$. Currently in S-NPP calibration, lunar observations have been used together with SDSM observations to address the angular dependent changes in the SD BRDF in order to provide an accurate monitoring of on-orbit changes in SD BRDF in the RTA SD view direction. With small changes at the mission beginning, the angular dependence can be ignored. 
The on-board BB provides TEB calibration source radiance. Its temperature is measured by 6 thermistors embedded in the BB panel on a scan-by-scan basis. The BB is nominally maintained at $292.6 \mathrm{~K}$ for both SNPP and N-20. Figure 4 illustrates on-board BB temperature short-term stability and uniformity for both VIIRS instruments, with continuous data over 3 orbits. The variation, within $25 \mathrm{mK}$, of the average temperature from day to night orbit is small and expected. At this controlled temperature, the N-20 BB shows a slightly larger standard deviation than S-NPP. Nevertheless, both instruments meet the uniformity design requirement of $30 \mathrm{mK}$. Their performance has been excellent at the nominal temperature setting.
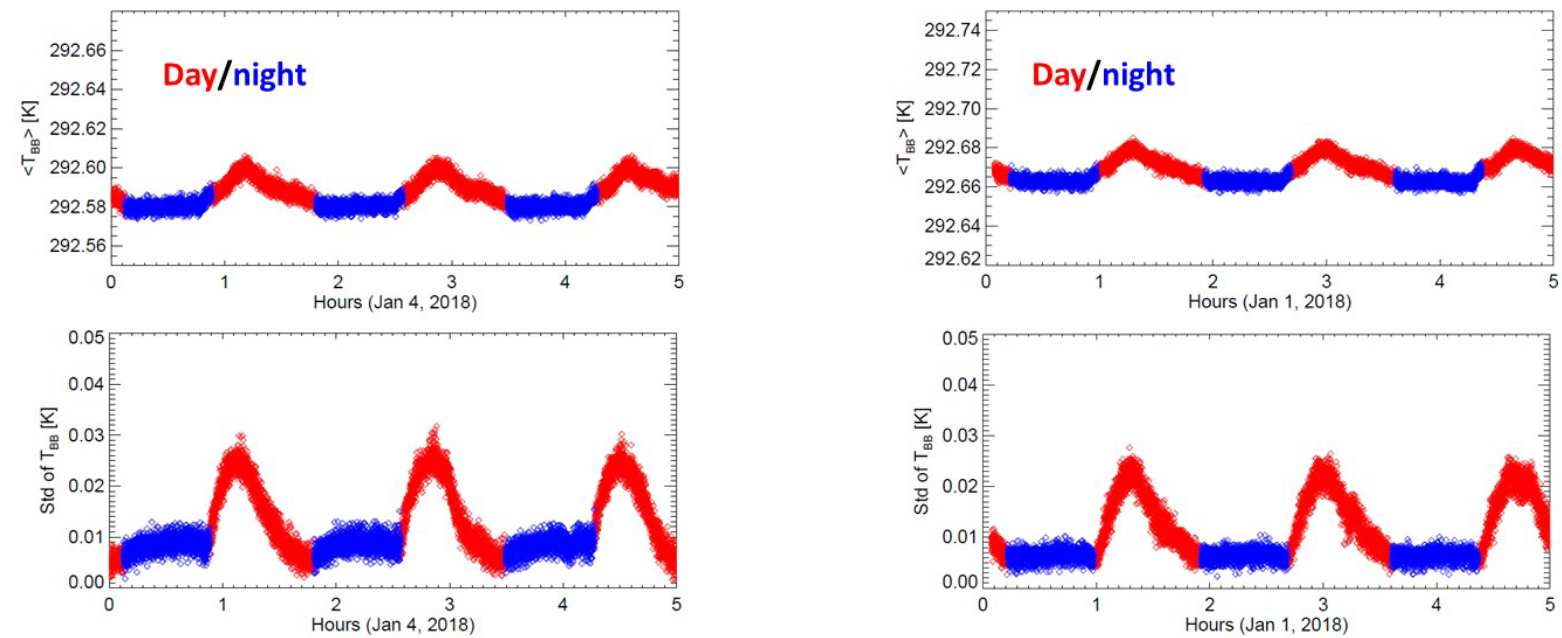

Figure 4. Blackbody scan-by-scan short-term (3 orbits) stability (upper plots) for N-20 (left) and S-NPP (right), and its uniformity or standard deviation (lower plots) among 6 thermistors.
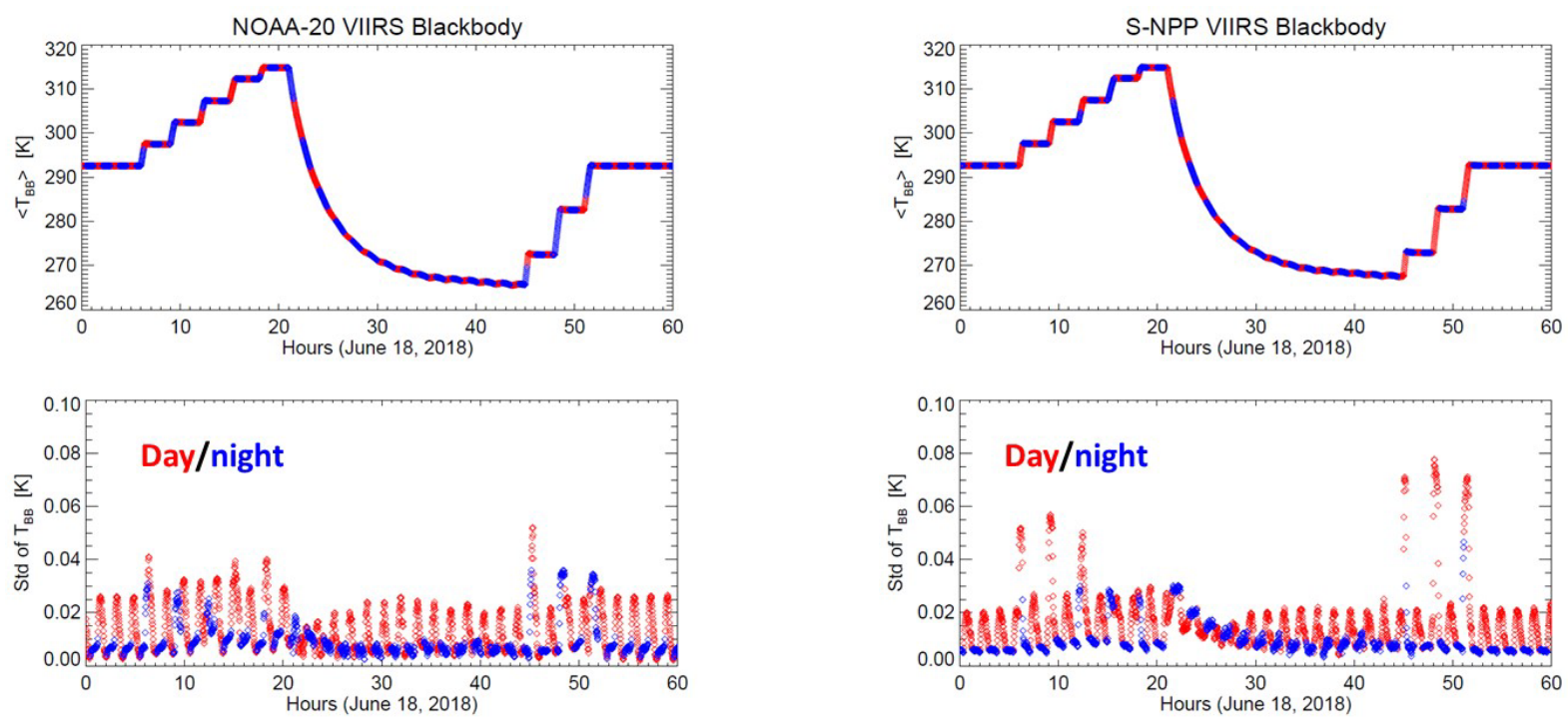

Figure 5. Blackbody temperature profiles (upper) and its uniformity or standard deviation (bottom) among 6 thermistors during the BB warm-up and cool-down for N-20 (left) and S-NPP (right).

Figure 5 shows examples of the quarterly BB WUCD performance for both VIIRS instruments. Each WUCD takes about 46 hours. The continuous cool-down data are used to calculate TEB detectors' offset and nonlinear coefficients, which have been stable from launch to present. The warm-up process includes 8 
temperature steps displayed in the upper plots. Data from different temperature plateaus and the lowest temperature at the end of cool-down are used to characterize detector noise performance. The bottom plots show the uniformity of the BB throughout the WUCD process. The non-uniformity is larger in the day time orbits and during warm-up, especially for S-NPP. During each warm-up period between two controlled plateaus, the non-uniformity exceeds the design requirement of $30 \mathrm{mK}$, resulting in lower SDR quality especially in LWIR on S-NPP.

Figure 6 shows the on-orbit gain change for the VIIRS VIS/NIR bands. The gains are very stable on-orbit for $\mathrm{N}-20$, with band M1 having an increase of up to $0.7 \%$ and all other bands having changes of $<0.4 \%$ in the first 250 days since launch. By contrast, the gain for S-NPP had significant changes over the same period, particularly for the NIR bands (up to $26 \%$ for bands M7 and I2), due to a contamination of the RTA mirrors which resulted in a rapid degradation in their reflectance $e^{5-7}$.
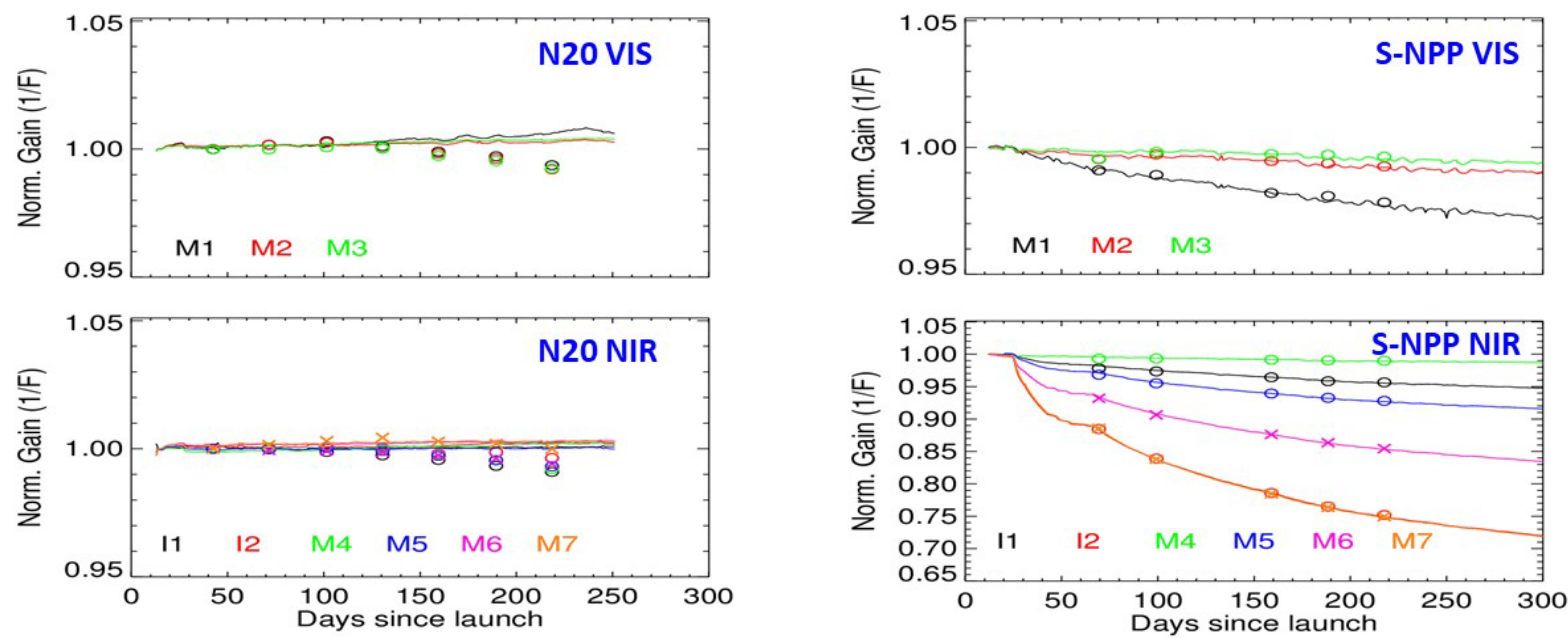

Figure 6. Normalized gain (inverse of F-factor) for N-20 (left) and S-NPP (right) VIS and NIR bands. Solid lines are SD measurements and symbols are lunar measurements.

The on-orbit gains derived from lunar observations are also displayed in Figure 6 and are generally in good agreement with the SD F-factors. The lunar-derived F-factors can have up to $1 \%$ seasonal oscillations largely due to different lunar libration angles; so the slight divergence of the SD and lunar F-factors for N-20 does not necessarily indicate a disagreement between the two calibration sources. The long-term agreement of the on-orbit gain from the SD and lunar measurements for N-20 will be carefully evaluated after at least one full year of lunar observations are completed. After a few years into the mission, the S-NPP VIIRS lunar gain was found to slightly deviate from the SD gain calculated using the H-factor derived at the SDSM viewing angle. Because of this, the long-term lunar gain trends are used to determine the SD H-factor at the view angle of the RTA. The current SD F-factors are calculated using the newly adjusted SD H-factors.

The SD observations at different illumination conditions, which produce different signal levels, are also used to characterize the RSB SNR ${ }^{15}$. Two sets of SNR values at typical radiances are listed in Table 1 for both SNPP and N-20: one at the beginning of each mission and another at a more recent date. The SNR is above the specification in all cases. It should be noted that the SNR values for S-NPP NIR and SWIR bands have decreased over the $6+$ years of operation. This is mainly due to the degradation of their responses (see Fig. 
6). The SNRs for N-20 RSB are about the same as or better than for S-NPP. For band M11, there was a change in the specified $\mathrm{L}_{\text {typ }}$ from 0.12 (S-NPP) to $1.0(\mathrm{~N}-20) \mathrm{W} / \mathrm{m}^{2} / \mu \mathrm{m} / \mathrm{sr}$, which is the primary reason that the M11 SNR for N-20 becomes much larger than for S-NPP.
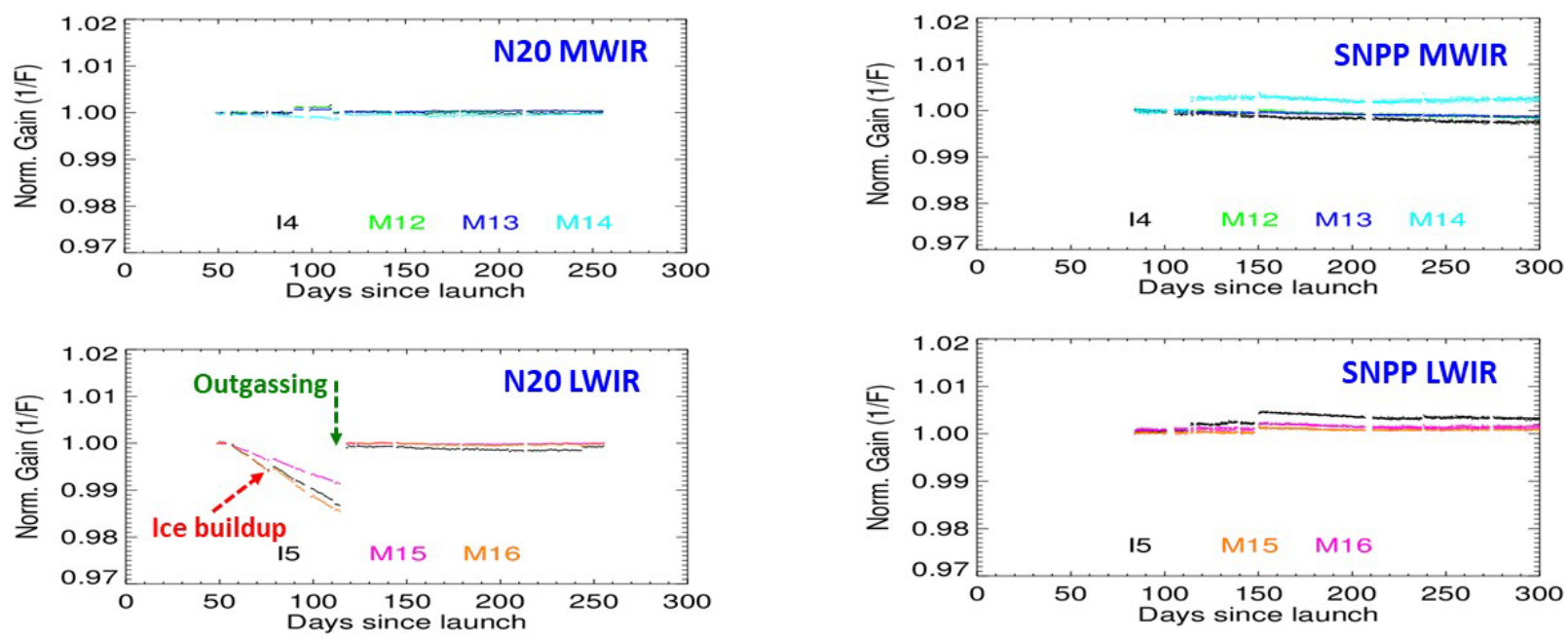

Figure 7. Normalized gain (inverse F-factor) for N-20 (left) and S-NPP (right) MWIR and LWIR bands (HAM-A).

The inverse of the F-factor (or gain) is used to assess the TEB detector on-orbit performance. This is not a direct SDR quality assessment, but demonstrates the stability of detectors' responses over time. The left panel of Figure 7 shows trends of the N-20 VIIRS TEB gains starting from Jan 6, 2018 (52 days into the mission) when the cold FPAs were controlled to their nominal operating temperature. The gain values for HAM-side A are averaged over all detectors within a band. In the case of S-NPP, the TEB gain over a similar timescale is plotted for comparison purposes (right panel of Fig. 7). In the case of MWIR bands, both instruments show a very small gain change to within $0.3 \%$ in the first 250 days of operation. After more than 6 years of on-orbit operation, the S-NPP MWIR gain change remains within $0.5 \%$. Similar performance is observed for the S-NPP LWIR bands. The changes in detector responses over the entire mission are within $0.3 \%$, except for band I5 that has experienced approximately $1.8 \%$ gain degradation to date. For N-20 LWIR, ice buildup on the LWIR FPA dewar window led to an accelerated gain change at mission beginning. A mid-mission outgassing performed on March 12, 2018 allowed the LWIR band gains to be restored close to their initial at launch values (within $0.3 \%$ ). As the TEB calibration is performed on a per-scan basis, the initial gain degradation has shown negligible impacts on the SDR quality. As expected, the HAM side B trend is similar to HAM side A. Also, the on-orbit TEB NEdT values remain nearly the same since launch, meeting the specified requirements.

Figure 8 shows examples of DNB daily averaged LGS F-factors (N-20: top left; S-NPP: top right), which are inversely proportional to the detector gains in unit of $\mathrm{W} \cdot \mathrm{cm}^{-2} \cdot \mathrm{sr}^{-1}$ for HAM side A and aggregation mode 1 . The trends of 16 detectors, plotted in different symbols/colors, are nearly identical for both N-20 and S-NPP. Also presented in Figure 8 (two bottom plots) are the corresponding DNB gain ratio trends (MGS/LGS), derived using the low-level signals from selected SD observations. Similar to the RSB, the N-20 DNB LGS F-factors and gain ratios (MGS/LGS and HGS/MGS) are very stable. The MGS/LGS ratios appear to be in two groups. This should not be surprising as different gain stages are aggregated using different detectors (pixels) in different CCD arrays. For S-NPP, the DNB F-factors at all three gain stages show similar trends, 
corresponding to the decrease of detector gains, and the gain ratios are nearly constant. This behavior is consistent with the degradation in the NIR spectral region as the DNB spectral bandwidth ranges from 0.5 to $0.9 \mu \mathrm{m}$. The plot timeline for N-20 is from the launch date $11 / 18 / 2017$ to $08 / 01 / 2018$, and for S-NPP is from launch date $10 / 28 / 2011$ to $08 / 01 / 2018$.
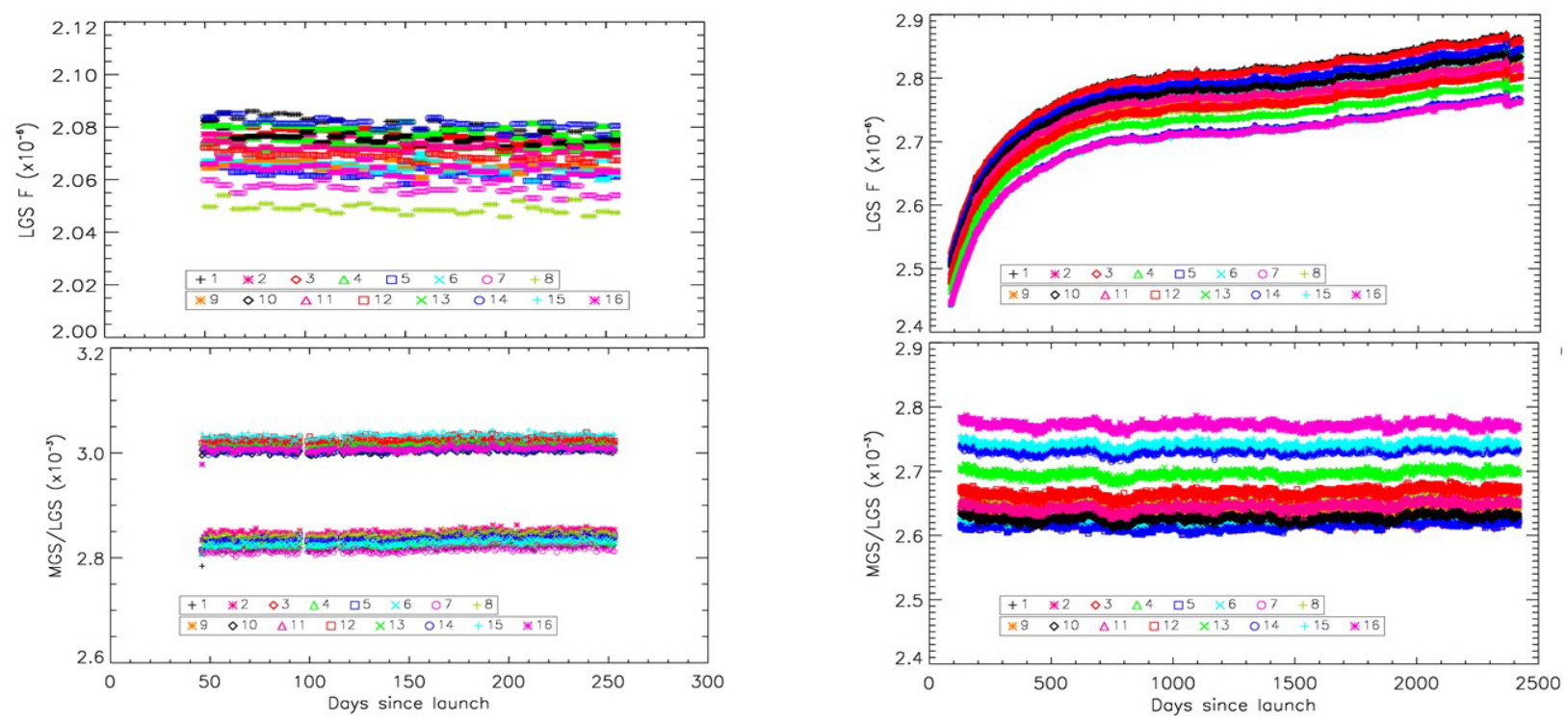

Figure 8. On-orbit trends of DNB daily averaged LGS F-factors (top left: N-20; top right: S-NPP) and gain ratios of MGS to LGS (bottom left: N-20; bottom right: S-NPP) for HAM side A and aggregation mode 1. Results of all 16 individual detectors are plotted with different symbols and colors.
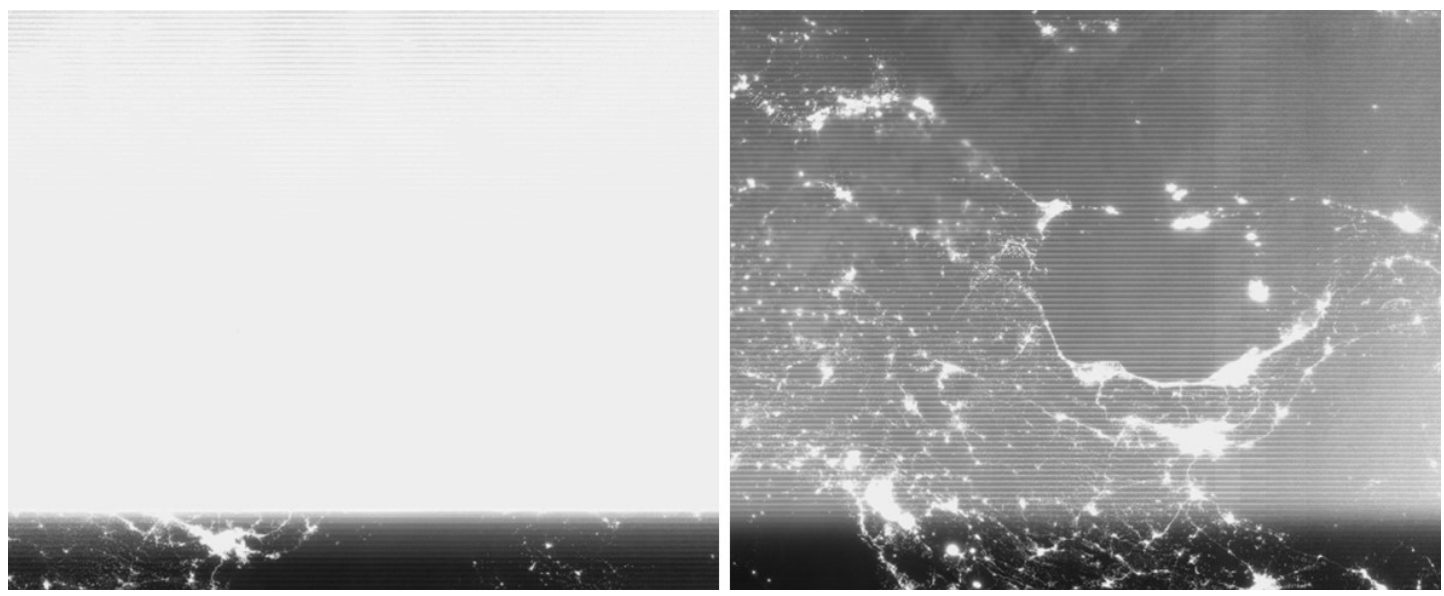

Figure 9. Left: S-NPP VIIRS DNB nighttime image over Europe (from granule 22:10:48, 07/13/2018) before stray light correction is applied. Right: N-20 VIIRS DNB nighttime image over Europe (from granule 22:59:37, $07 / 13 / 2018$ ) before stray light correction is applied.

Figure 9 shows two VIIRS DNB nighttime images over Europe acquired on July 13, 2018, one from S-NPP and another from N-20 data granules of approximately 50 min apart. Most areas of the selected images are impacted by the stray light contamination. The same images after the stray light correction are illustrated in Figure 10. Currently, the same DNB stray light correction algorithms are applied for both S-NPP and N-20 
with their correction coefficients derived independently ${ }^{11}$. The corrections for both instruments have been very effective. In order to reduce the detector nonlinear effect in N-20 DNB high aggregation zones, a different aggregation configuration (option 21) is applied, resulting in extended area coverage. In general, the N-20 DNB stray light contamination is smaller than S-NPP. The stray light contamination in the extended area in $\mathrm{N}-20$, however, is much higher than other areas and requires more effort when deriving the correction coefficients.
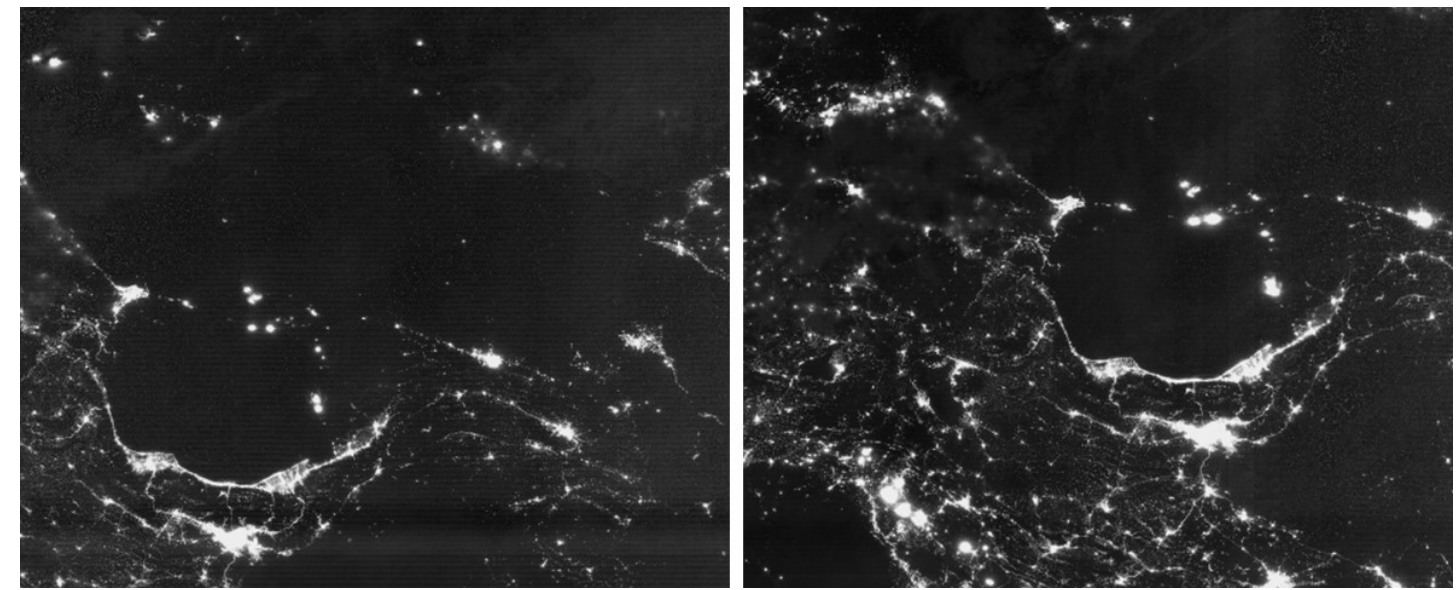

Figure 10. Same as Figure 9 but after stray light correction is applied.
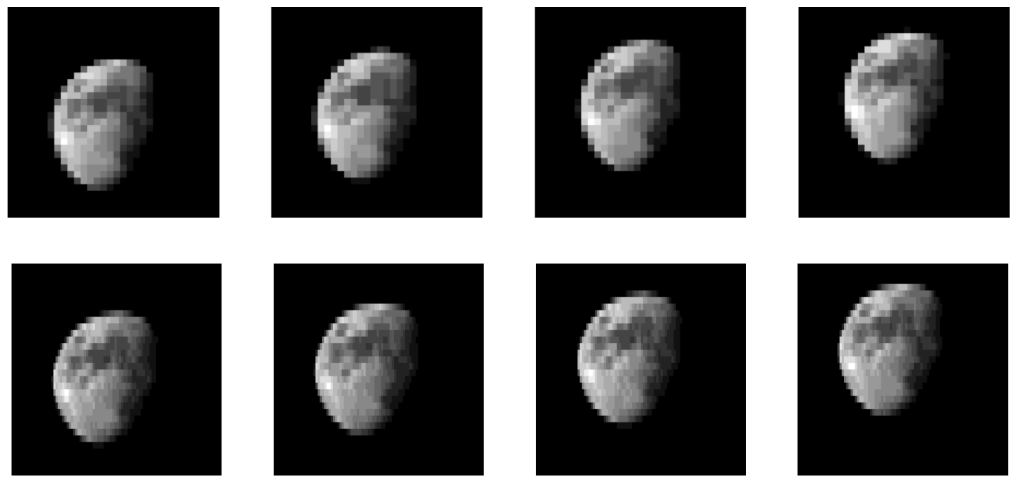

Figure 11. VIIRS I1 lunar images from calibration events on April 25, 2018 (upper/lower panel: S-NPP/N-20).

The same lunar calibration strategy has been applied for both VIIRS instruments. Their lunar observations are normally made on the same day (50 min apart). Lunar observations are used primarily to monitor RSB calibration stability (see Figure 6). Meanwhile, the lunar images can also be used to characterize the band-toband registration (BBR). The lunar BBR approach was initially developed for MODIS and validated using one of its on-board calibrators that has the capability to perform on-orbit spatial characterization ${ }^{16}$. Shown in Figure 11 are the band I1 lunar images (middle four scans) taken from the S-NPP and N-20 lunar calibration events on April 25, 2018. Figure 12 shows the along-scan BBR for S-NPP and N-20 at their mission beginning and that in June 2018. For both instruments, the on-orbit changes in BBR are very small (within 0.05 M-band pixel size) for both along-scan and along-track directions. 
Lunar observations have also been used to examine N-20 and S-NPP calibration consistency. Preliminary lunar calibration inter-comparison results derived using the official operational F-factors indicate 2-3\% calibration differences between the two sensors' RSB. Similar results have also been derived using other calibration inter-comparison approaches. Clearly, more efforts are needed in future studies to fully address the calibration consistency between the two VIIRS instruments.
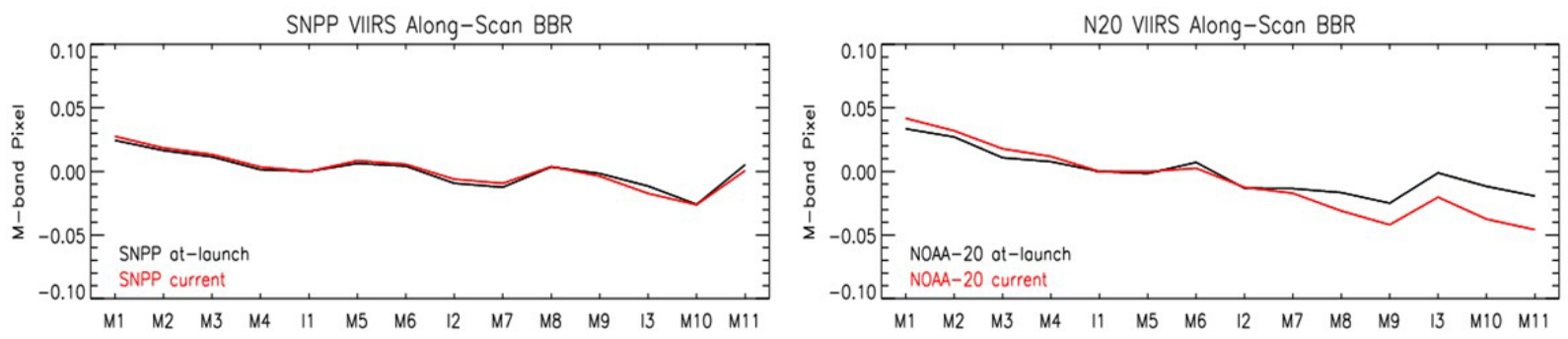

Figure 12. S-NPP and N-20 VIIRS Along-scan BBR from lunar measurements. At launch values shown in black and current values (June 2018) values shown in red.

\section{SUMMARY}

This paper has provided an overview of N-20 VIIRS on-orbit performance of the first eight months and comparisons to the S-NPP VIIRS early mission performance. In general, the N-20 VIIRS performance is at least as good as or better than the S-NPP VIIRS in the same time period since their respective launches. The N-20 RSB on-orbit responses have very stable compared to S-NPP and have shown no sign of the mirror contamination that led to the rapid degradation of the S-NPP NIR and SWIR bands. The N-20 ICV analysis was able to quickly identify an ice buildup problem affecting the performance of some TEB. A mid-mission outgassing was able to remove the problem and restore the gains for the affected TEB. All the TEB now show stable trends with no sign of on-orbit degradation, similar to S-NPP. Like the RSB, the N-20 DNB gains have had much more stable on-orbit performance than S-NPP, and in addition the N-20 DNB has so far been less affected by stray light. Other N-20 initial calibration and characterization activities were also performed and did not identify any serious concerns.

The performance in the first eight months of operation is a good indication that the N-20 will have less overall degradation in the long-term than S-NPP. More detailed evaluations and comparisons to S-NPP VIIRS will be possible after the N-20 VIIRS completes at least a full yearly cycle. For example, the relative comparison of the RSB gains derived from the lunar data and the SD data will be evaluated after a couple of years in order to determine if any adjustment is needed to the SD calibration, as was done for S-NPP.

\section{ACKNOWLEDGEMENTS}

The authors would like to acknowledge other members of the VIIRS Characterization Support Team (VCST) for their contributions and support.

\section{REFERENCES}

1. Schueler, C. F., E. Clement, P. Ardanuy, C. Welsh, F. De Luccia, and H. Swenson, "NPOESS VIIRS sensor design overview," Proc. SPIE, 4483, 11-23, 2002 
2. Murphy, R.P., P. E. Ardanuy, F. Deluccia, J. E. Clement, and C. Schueler, "The visible infrared imaging radiometer suite, Earth Science Satellite Remote Sensing,” vol. 1, New York, USA: Springer-Verlag, pp. 199-223, 2006

3. Xiong X., J. Butler, K. Chiang, B. Efremova, J. Fulbright, N. Lei, J. McIntire, H. Oudrari, J. Sun, Z. Wang, and A. Wu, "VIIRS On-orbit Calibration Methodology and Performance," JGR Vol. 119, Issue 9, pp 5065-5078, 2014

4. Cao, C., F. Deluccia, X. Xiong, R. Wolfe, and F. Weng, "Early On-orbit Performance of the Visible Infrared Imaging Radiometer Suite (VIIRS) onboard the Suomi National Polar-orbiting Partnership (SNPP) Satellite," IEEE Trans. Geosci. Remote Sens., vol. 52, no.2, pp.1142-1156, doi: 10.1109/TGRS.2013.2247768, 2014

5. De Luccia, F., D. Moyer, E. Johnson, K. Rausch, K., N. Lei, K. Chiang, X. Xiong, J. Fulbright, E. Hass, and G. Iona, "Discovery and characterization of on-orbit degradation of the Visible Infrared Imaging Radiometer Suite (VIIRS) Rotating Telescope Assembly (RTA)," Proc. SPIE, vol. 8510, 85101A, 2012

6. Barrie, J., P. D. Fuqua, M. J. Meshishnek, M. Ciofalo, C. Chu, J. Chaney, R.Moision, and L. Graziani, "Root cause determination of on-orbit degradation of the VIIRS rotating telescope assembly," Proc. SPIE, 8510, 851009, 2012

7. Iona, G., J. J. Butler, B. Guenther, E. H. Johnson, B. Kennedy, C. J. Kent, R. W. Lambeck, E. Waluschka, and X. Xiong, "VIIRS on-orbit optical anomaly: Investigation, analysis, root cause determination and lessons learned," Proc. SPIE, 8510, 85101C, 2012

8. Fulbright, J., N. Lei, B. Efremova, and X. Xiong, "Suomi-NPP VIIRS Solar Diffuser Stability Monitor Performance,” IEEE Trans. Geosci. Remote Sens., vol. 54, 631-639, 2016, DOI: 10.1109/TGRS.2015.2441558

9. Lei, N., and X. Xiong, "Suomi NPP VIIRS Solar Diffuser BRDF Degradation Factor at Short-Wave Infrared Band Wavelengths", IEEE Trans. Geosci. Remote Sens., vol 54, issue 10, pp 6212-6216, 2016

10. Lee, S., J. McIntire, H. Oudrari, T. Schwarting, X. Xiong, "A New Method for Suomi-NPP SIIRS DayNight Band On-Orbit Radiometric Calibration,” IEEE Trans. Geosci. Remote Sens., vol. 53, 324-334, 2015

11. Chen, H., X. Xiong, C. Sun, X. Chen and K. Chiang, "Suomi-NPP VIIRS day-night band on-orbit calibration and performance," Journal of Applied Remote Sensing, vol 11 (3), 36019, 2017

12. Efremova, B., J. Mcintire, D. Moyer, A. Wu, and X. Xiong, "S-NPP VIIRS thermal emissive bands onorbit calibration and performance," Journal of Geophysical Research, Volume 119, Issue 18, pp 1085910875,2014

13. Xiong, X., J. Sun, J. Fulbright, Z. Wang, and J. Butler, "Lunar Calibration and Performance for S-NPP VIIRS Reflective Solar Bands," IEEE Trans. Geosci. Remote Sens., vol 54, 1052-1061, 2015

14. Xiong, X., J. Fulbright, A. Angal, Z. Wang, X. Geng, and J. Butler, "Assessment of MODIS and VIIRS solar diffuser on-orbit degradation," Proc. SPIE 9607, 96071T, 2015.

15. Twedt, K., N. Lei, and X. J. Xiong, "On-orbit noise characterization of the S-NPP VIIRS reflective solar bands," Proc. SPIE, 10402, 104021X, 2017

16. Wang, Z., X. Xiong, Y. Li, "Update of VIIRS on-orbit spatial parameters characterized with the Moon," IEEE Trans. Geosci. Remote Sens., vol. 53, 5486-5494, 2015 